\title{
A Calcitonin-Secreting Tumor of the Pancreas
}

\author{
Maurizio lacobone \\ Endocrine Surgery Unit, Department of Surgical and Gastroenterological Sciences, University of Padua, \\ Padua, Italy
}

We read with great interest the article 'Calcitonin-Secreting Tumor of the Pancreas' by Mullerpatan et al. [1]. The authors described a rare case of calcitonin-secreting neuroendocrine tumor of the head of the pancreas in a woman presenting with watery diarrhea, hypokalemia, hypercalcemia and hypercalcitoninemia. The patient also had undetectable preoperative intact $\mathrm{PTH}$ and ACTH levels. The tumor demonstrated immunoreactivity to calcitonin and (to a lesser extent) VIP, but it was negative to somatostatin. The postoperative period was characterized by profound hypocalcemia needing calcium and vitamin $\mathrm{D}$ replacement, by normalization of calcitonin, ACTH and intact PTH levels; TSH levels had a mild increase. The authors suggested that the decrease in calcitonin levels and increase of intact PTH, ACTH and TSH to normal levels might be the result of the removal of the calcitonin (or somatostatin) source and of their suppressive effects (negative feedback). In our opinion, some remarks are required.

It is not possible to suppose any biologic effects by a somatostatin hypersecretion, since somatostatin plasmatic measurements were not available and there was no evidence of histopathological immunoreactivity to somatostatin in the pancreatic tumor. In addition, it is not possible to explain hypercalcemia associated to undetectable intact PTH levels by hypercalcitoninemia. Although calcitonin could have potent effects in calcium metabolism, its role in humans is still unknown, as was also remarked by the authors. In experimental conditions, the hormone reduces blood calcium levels; in clinical conditions, usually (as in metastatic medullary thyroid carcinoma with high calcitonin plasmatic levels), hypercalcitoninemia is not associated to a significant variation of calcemia, intact PTH, ACTH and TSH levels. On the contrary, hypercalcemia (associated with undetectable intact PTH levels) should be considered the result of suppression of a normal parathyroid function (negative feedback).

In these conditions, hypercalcemia might be the consequence of bone metastases, or, rarely, of the ectopic production of PTH-like hormones (rpPTH, i.e. PTH-related protein), configuring a paraneoplastic syndrome. rpPTH is not measured by the specific assays for iPTH (i.e., normal intact PTH), that, subsequently, is suppressed.

It was the case of a patient that we have recently observed: a women with multinodular goiter, hypercalcitoninemia (>6,000 pg/ml), hypercalcemia, hypokalemia and watery diarrhea. Because of the suspicion of medullary thyroid carcinoma, the patient underwent a fine-needle aspiration biopsy. Cytology did not reveal malignancy, thus a pentagastrin-stimulation test was performed. Stimulated pentagastrin calcitonin had only a slight and not significant increase, as usually occurs in cases of extrathyroidal hypercalcitoninemia [2]. For this reason, a total body CT scan was performed and a 3-cm mass of the tail of the pancreas was found. In addition, intact PTH levels were not detectable, while high levels of rpPTH (PTH-related protein) were present. A distal pancreatectomy removed a malignant pancreatic neuroendocrine tumor with immunoreactivity to calcitonin and to VIP. After surgery, calcitonin, rpPTH and calcium plasmatic levels normalized.

Although extremely rare, neuroendocrine pancreatic tumors can produce a lot of hormones, with subsequent paraneoplastic syndromes. Hypercalcemia might be the result of the hyperproduction of rpPTH. This hormone is not measured by the usual specific assays for iPTH, that, subsequently, is suppressed because of hypercalcemia. In addition, we think that, since the most common cause of hypercalcitoninemia is metastatic medullary thyroid carcinoma, a pentagastrin stimulation test should be systematically performed in order to exclude this possibility [3].

\section{References}

1 Mullerpatan PM, Joshi SR, Shah RC, Tampi CS, Doctor VM, Jagannath P, Modlin I: Calcitonin-secreting tumor of the pancreas. Dig Surg 2004;21:321-324.

2 Machens A, Haedecke J, Holzhausen HJ, Machens A, Haedecke J, Holzhausen HJ, Thomusch O, Schneyer U, Dralle H: Differential diagnosis of calcitonin-secreting neuroendocrine carcinoma of the foregut by pentagastrin stimulation. Langenbecks Arch Surg 2000;385: 398-401.

3 Leboulleux S, Baudin E, Young J, Caillou B, Lazar V, Pellegriti G, Ducreux M, Schaison G, Schlumberger M: Gastroenteropancreatic neuroendocrine tumor metastases to the thyroid gland: Differential diagnosis with medullary thyroid carcinoma. Eur J Endocrinol 1999; 140:187-191.

\section{KARGER}

Fax +4161306 1234 E-Mailkarger@karger.ch www.karger.com
Maurizio Iacobone, MD

Endocrine Surgery Unit, Department of Surgical and Gastroenterological Sciences University of Padua, Via Giustiniani, 2

IT-35128 Padova (Italy)

Tel. +39049 8212388, Fax +390498211378, E-Mail maurizio.iacobone@unipd.it 